\title{
CORRELATION OF HEMOPHILIA JOINT HEALTH SCORE (HJHS) AND FACTOR VIII INHIBITOR IN CHILDREN WITH HEMOPHILIA A AT DR SOETOMO HOSPITAL
}

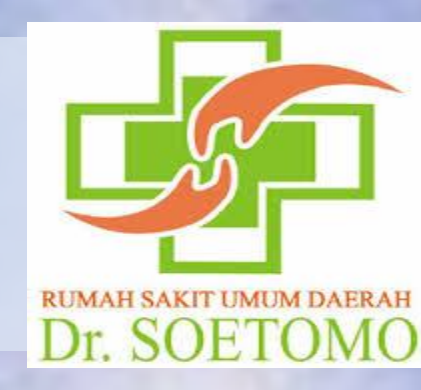

Ajeng Indriastari, Andi Cahyadi ${ }^{1}$, Maria C Shanty ${ }^{1}$, Mia Ratwita Andarsini ${ }^{1}, \quad$ IDG Ugrasena ${ }^{1}$, Bambang Permono ${ }^{1}$, Ratna D Haryadi ${ }^{2}$, Endang Retnowati ${ }^{3}$, Fery H Soedewo ${ }^{3}$

Department of Child Health ${ }^{1}$, Department of Physical Medicine and Rehabilitation ${ }^{2}$, Department of Clinical Pathology ${ }^{3}$ Medical School,Airlangga University, Dr. Soetomo Hospital, Surabaya, Indonesia

\section{BACKGROUND}

Joint bleeding is the most common bleeding manifestation in hemophilia A. The Hemophilia Joint Health Score (HJHS) is a scoring tool to detect early stage of hemophilic arthropathy and to evaluate progressivity of hemophilia. There were no available data of the association between HJHS and factor VIII inhibitor in Indonesia.

\section{OBJECTIVE}

To evaluate the correlation between HJHS and factor VIII inhibitor in children with Hemophilia.

\section{METHODS}

A cross-sectional study was conduct on children 4-18 years with hemophilia A at pediatric hematology oncology out patient department Dr. Soetomo Hospital, June-July 2016. HJHS and factor VIII inhibitor examination were performed. Data analysis was performed using Spearman test.

\section{RESULTS}

A total of 29 cases were enrolled. Mean Age was 10,2 (SD 3,93) years (Ranged 4-18 years)

Table 1. Patients Characteristic

\begin{tabular}{|c|c|c|}
\hline Characteristic & $\mathbf{n}$ & $\%$ \\
\hline \multicolumn{3}{|l|}{ Age Group } \\
\hline 3-6 years & 6 & 20.7 \\
\hline $6-12$ years & 11 & 37.9 \\
\hline $12-20$ years & 12 & 41.4 \\
\hline \multicolumn{3}{|l|}{ Degree of Hemophilia } \\
\hline Mild & 7 & 24.1 \\
\hline Moderate & 16 & 55.2 \\
\hline Severe & 6 & 20.7 \\
\hline \multicolumn{3}{|l|}{ Type of bleeding } \\
\hline Hemarthrosis & 12 & 41.4 \\
\hline \multicolumn{3}{|l|}{ Non-Hemarthrosis } \\
\hline - Intracranial bleeding & 3 & 10.3 \\
\hline - Gastrointestinal bleeding & 3 & 10.3 \\
\hline - Mucosal bleeding & 3 & 10.3 \\
\hline - Post circumcision & 8 & 27.7 \\
\hline \multicolumn{3}{|l|}{ Hemarthrosis Location } \\
\hline Elbow & 7 & 24.1 \\
\hline Knee & 13 & 44.9 \\
\hline Ankle & 9 & 31 \\
\hline \multicolumn{3}{|l|}{ Type of Hemarthrosis } \\
\hline Spontaneous & 27 & 93.1 \\
\hline Traumatic & 2 & 6.9 \\
\hline \multicolumn{3}{|l|}{ Frequency of Hemarthrosis / month } \\
\hline$<3 x$ & 13 & 44.9 \\
\hline $3-5 x$ & 9 & 31 \\
\hline $5-10 x$ & 6 & 20.7 \\
\hline$>10 x$ & 1 & 3.4 \\
\hline \multicolumn{3}{|l|}{ Joint Deformity } \\
\hline Yes & 16 & 55.1 \\
\hline No & 13 & 44.9 \\
\hline \multicolumn{3}{|l|}{ Mobility } \\
\hline Unlimited & 8 & 27.6 \\
\hline Recreational limitations & 18 & 62.1 \\
\hline School limitations & 1 & 3.4 \\
\hline Mobility equipment & 2 & 6.9 \\
\hline
\end{tabular}

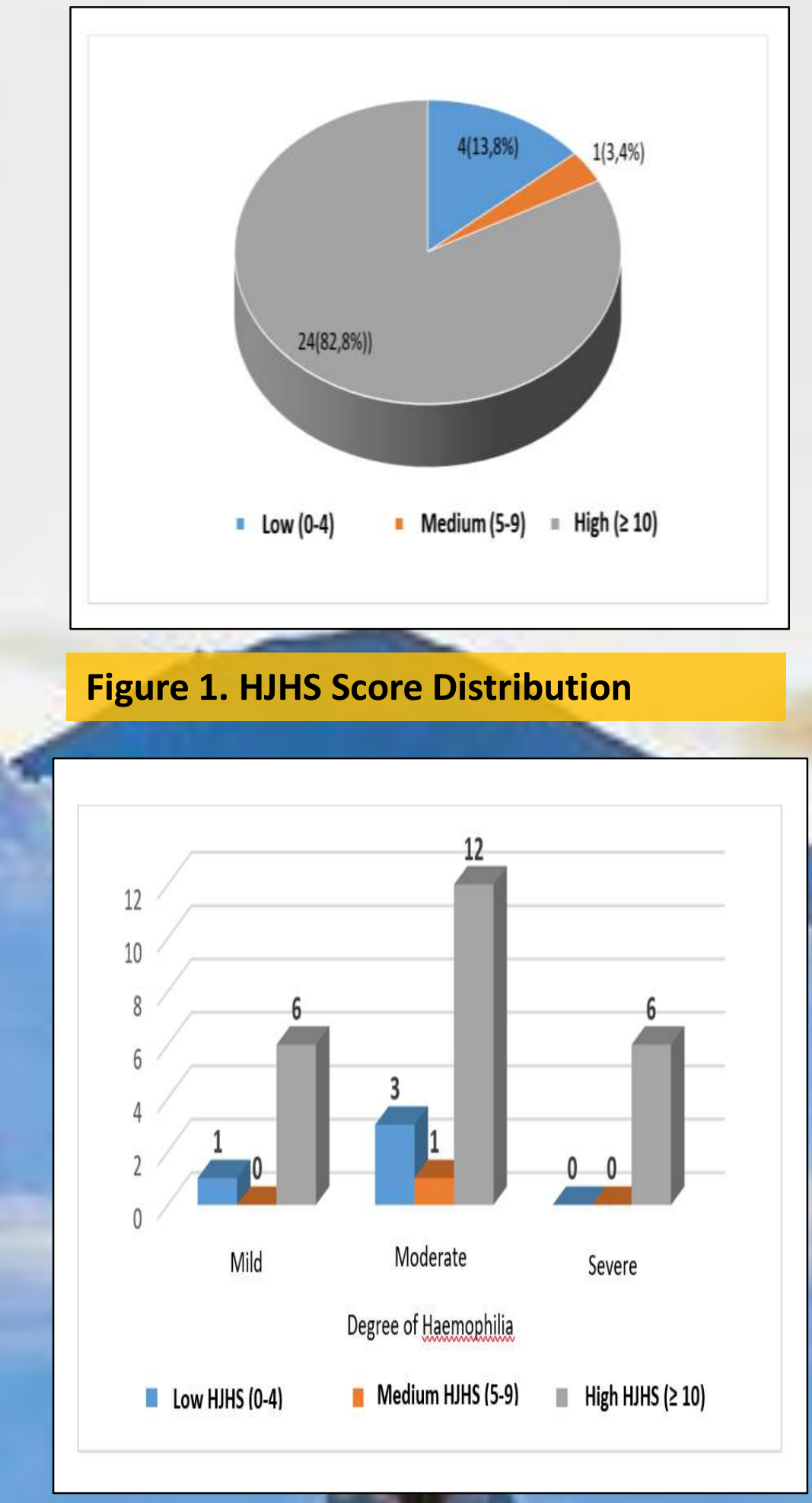

Figure 2. HJHS Score Distribution and Degree of Haemophilia

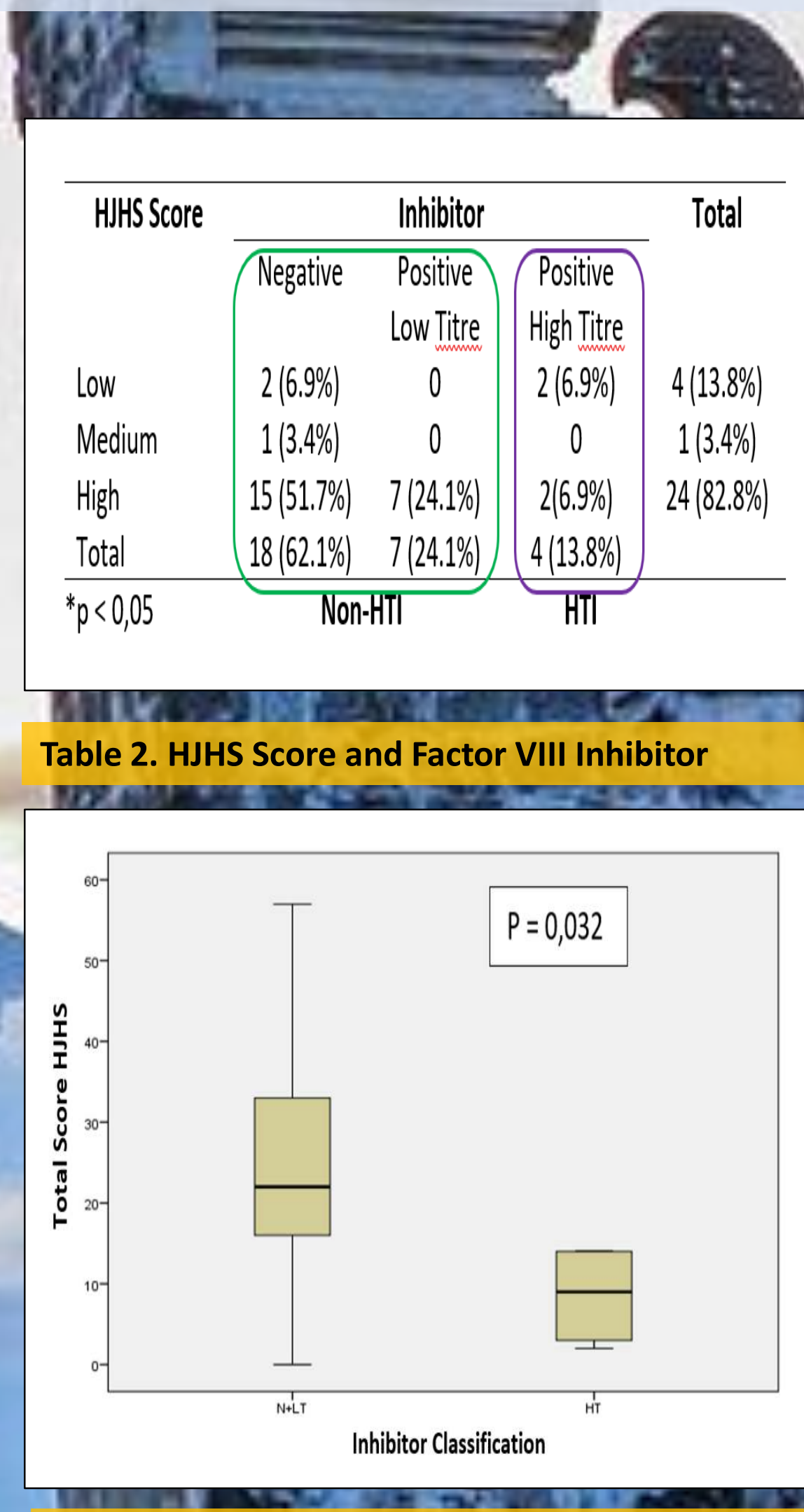

Figure 3. Correlation between HJHS Score and Factor VIII Inhibitor 